\title{
Defining Potential Overutilization of Physical Therapy Consults on Hospital Medicine Services
}

\author{
Maylyn Martinez, MD*, Matthew Cerasale, MD, MPH${ }^{1}$, Mahnoor Baig $^{2}$, Claire Dugan, MD³, \\ Marla Robinson, MSc, OTR/L ${ }^{4}$, Meghan Sweis, MSN5, Micah Prochaska, MD, MS ${ }^{1}$, Andrew Schram, MD, MBA', \\ David Meltzer, MD, PhD', Vineet M Arora, MD, MAPP3
}

\begin{abstract}
${ }^{1}$ Section of Hospital Medicine, Department of Medicine, University of Chicago, Chicago, Illinois; ${ }^{2}$ University of Illinois, Chicago, Illinois; ${ }^{3}$ Section of General Internal Medicine, Department of Medicine, University of Chicago, Chicago, Illinois; ${ }^{4}$ Inpatient Therapy Services Department, University of Chicago, Chicago, Illinois; ${ }^{5}$ Department of Nursing Administration and Patient Services, University of Chicago, Chicago, Illinois.
\end{abstract}

Appropriate use of inpatient physical therapy services is important for preventing hospital-associated disability (HAD). We assessed potential overutilization of physical therapy consults on hospital medicine services using the Activity Measure-Post Acute Care (AM-PAC) score. Our sample included 3592 unique admissions (mean age, 66 years; $48 \%$ women) at a large academic medical center. Based on an AM-PAC cutoff of $>43.63$ (raw score, 18) in patients who were discharged to home, $38 \%$ of physical therapy consults were considered "potential overutilization."
Combined with age $<65$ years, $18 \%$ of consults remained "potential overutilization." After adjustment for age, sex, and length of stay, patients admitted with high mobility scores were 5.38 times more likely to be discharged to home (95\% Cl, 4.36-2.89) compared with those with low mobility scores. Being more judicious with physical therapy consults and reserving skilled therapy for at-risk patients could help prevent HAD while also having a positive impact on healthcare systems. Journal of Hospital Medicine 2021;16:553-555. (c) 2021 Society of Hospital Medicine

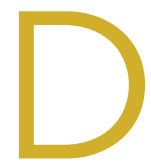
uring hospitalization, patients spend $87 \%$ to $100 \%$ of their time in bed. ${ }^{1}$ This prolonged immobilization is a key contributor to the development of hospital-associated disability (HAD), defined as a new loss of ability to complete one or more activities of daily living (ADLs) without assistance after hospital discharge. HAD can lead to readmissions, institutionalization, and death and occurs in approximately one-third of all hospitalized patients. ${ }^{2,3}$ The most effective way to prevent HAD is by mobilizing patients early and throughout their hospitalization. ${ }^{4}$ Typically, physical therapists are the primary team members responsible for mobilizing patients, but they are a constrained resource in most inpatient settings.

The Activity Measure-Post Acute Care Inpatient Mobility Short Form (AM-PAC IMSF) is a validated tool for measuring physical function. ${ }^{5}$ The AM-PAC score has been used to predict discharge destination within 48 hours of admission 6 and as a guide to allocate inpatient therapy referrals on a medical and a neurosurgical service. ${ }^{7,8}$ To date, however, no studies have used AM-PAC scores to evaluate overutilization of physical therapy consults on direct care hospital medicine

*Corresponding Author: Maylyn Martinez, MD;

Email: maylynm@medicine.bsd.uchicago.edu; Telephone: 773-702-7615;

Twitter: @MS_Martinez_MD.

Published online first August 18, 2021.

Received: March 26, 2021; Revised: June 21, 2021; Accepted: June 24, 2021

(C) 2021 Society of Hospital Medicine DOI 10.12788/jhm.3673 services. In this study, we aimed to assess the potential overutilization of physical therapy consults on direct care hospital medicine services using validated AM-PAC score cutoffs.

\section{METHODS}

\section{Study Design and Setting}

We analyzed a retrospective cohort of admissions from September 30, 2018, through September 29, 2019, on all direct care hospital medicine services at the University of Chicago Medical Center (UC), Illinois. These services included general medicine, oncology, transplant (renal, lung, and liver), cardiology, and cirrhotic populations at the medical-surgical and telemetry level of care. All patients were hospitalized for longer than 48 hours. Patients who left against medical advice; died; were discharged to hospice, another hospital, or an inpatient psychiatric facility; or received no physical therapy referral during admission were excluded. For the remaining patients, we obtained age, sex, admission and discharge dates, admission and discharge AM-PAC scores, and discharge disposition.

\section{Mobility Measure}

At UC, the inpatient mobility protocol requires nursing staff to assess and document AM-PAC mobility scores for each patient at the time of admission and every nursing shift thereafter. They utilize the original version of the AM-PAC "6-Clicks" Basic Mobility score, which includes three questions assessing difficulty with mobility and three questions assessing help needed with mobility activities. It has high interrater reliability, with an intraclass correlation coefficient of 0.85 .9 


\section{Outcomes and Predictors}

The primary outcome was "potential overutilization." Secondary outcomes were discharge disposition and change in mobility. Our predictors included admission AM-PAC score, age, and sex. Based on previous studies that validated an AM-PAC score of 42.9 (raw score, 17) as a cutoff for predicting discharge to home, ${ }^{6}$ we defined physical therapy consults as "potentially inappropriate" in patients with admission AM-PAC scores $>43.63$ (raw score, 18) who were discharged to home. Likewise, in the UC mobility protocol, nursing staff independently mobilize patients with AM-PAC scores $>18$, another rationale to use this cutoff for defining physical therapy consult inappropriateness. "Discharge to home" was defined as going home with no additional needs or services, going home with outpatient physical therapy, or going home with home health physical therapy services, since none of these require inpatient physical therapy assessment for the order to be placed. Discharge to longterm acute care, skilled nursing facility, subacute rehabilitation facility, or acute rehabilitation facility were considered "discharge to post-acute care." Loss of mobility was calculated as: discharge AM-PAC - admission AM-PAC, termed delta AM-PAC.

\section{Statistical Analysis}

Descriptive statistics were used to summarize age (mean and $\mathrm{SD}$ ) and age categorized as $<65$ years or $\geq 65$ years, sex (male or female), admission AM-PAC score (mean and SD) and categorization ( $\leq 43.63$ or $>43.63$ ), discharge AM-PAC score (mean and SD), and discharge destination (home vs post-acute care). Chi-square analysis was used to test for associations between admission AM-PAC score and delta AM-PAC. Two-sample t-test was used to test for difference in mean delta AM-PAC between admission AM-PAC groups. Multivariable logistic regression was used to test for independent associations between age, sex, and admission AM-PAC score and odds of being discharged to home, controlling for length of stay. $P$ values of $<.05$ were considered statistically significant for all tests. Analyses were performed using Stata statistical software, release 16 (StataCorp LLC).

\section{RESULTS}

During the 1-year study period, 3592 admissions with physical therapy consults occurred on the direct care hospital medicine services (58\% of all admissions). Mean age was 66.3 years (SD, 15.4 years), and $48 \%$ of patients were female. The mean admission AM-PAC score was 43.9 (SD, 11.1), and the mean discharge AM-PAC score was 46.8 (SD, 10.8). In our sample, $38 \%$ of physical therapy consults were for patients with an AM-PAC score $>43.63$ who were discharged to home and were therefore deemed "potential overutilization." Of those, $40 \%$ were for patients who were 65 years or younger ( $18 \%$ of all physical therapy consults) (Table 1).

A higher proportion of patients with AM-PAC scores $>43.63$ were discharged to home compared with those with AM-PAC scores $\leq 43.63$ (89\% vs 55\%; $\chi^{2}[1, N=3099]$, 396.5; $P<.001$ ). More patients younger than 65 years were discharged to home compared with those 65 years and older (79\% vs 63\%; $\left.\chi^{2}[1, N=3099], 113.6 ; P<.001\right)$. Additionally, for all patients
TABLE 1. Sample Characteristics

\begin{tabular}{|c|c|}
\hline \multicolumn{2}{|l|}{$\begin{array}{l}\text { Participants } \\
(\mathrm{N}=3592)\end{array}$} \\
\hline Age, mean (SD), y & $66.3(15.4)$ \\
\hline \multicolumn{2}{|l|}{ Age groups, \% } \\
\hline$<65 y$ & 43 \\
\hline$\geq 65$ y & 57 \\
\hline Female, \% & 48 \\
\hline Admission AM-PAC score, mean (SD) & $43.9(11.1)$ \\
\hline Discharge AM-PAC score, mean (SD) & $46.8(10.8)$ \\
\hline High admission AM-PAC, \% & 42 \\
\hline High admission AM-PAC and discharged to home, \% & 38 \\
\hline All discharged to home, \% & 70 \\
\hline High admission AM-PAC, \% & $89^{a}$ \\
\hline Low admission AM-PAC, \% & $55^{a}$ \\
\hline Age $<65 y, \%$ & $79^{a}$ \\
\hline Age $\geq 65$ y, $\%$ & $63^{a}$ \\
\hline Age $<65 y+$ high AM-PAC + discharge to home, $\%$ & 18 \\
\hline \multicolumn{2}{|l|}{ Delta AM-PAC } \\
\hline All, mean (SD) & $3.15(8.79)$ \\
\hline High admission AM-PAC (95\% CI) & $-0.41(0.82-0.01)^{b}$ \\
\hline Low admission AM-PAC (95\% Cl) & $5.69(5.28-6.09)^{b}$ \\
\hline \multicolumn{2}{|l|}{ Negative delta AM-PAC, \% } \\
\hline High admission AM-PAC & $34^{a}$ \\
\hline Low admission AM-PAC & $21^{\mathrm{a}}$ \\
\hline \multicolumn{2}{|c|}{$\begin{array}{l}\text { High admission AM-PAC score is defined as }>43.63 \text {; low admission AM-PAC score is defined as } \\
\leq 43.63 \text { based on a previous study showing a score of } 42.9 \text { for AM-PAC basic mobility predicts } \\
\text { discharge to home. }{ }^{6} \text { A T-scale score of } 43.63 \text { corresponds to a raw score of } 18 \text {. }\end{array}$} \\
\hline \multicolumn{2}{|c|}{ association by chi-square test, $P<.001$} \\
\hline \multicolumn{2}{|l|}{${ }^{b}$ Difference in means by two-sample $t$-test } \\
\hline Abbreviation: AM-PAC, Activity Measure-Post Acute Ca & \\
\hline
\end{tabular}

younger than 65 years, those with AM-PAC score $>43.63$ were discharged to home more frequently than those with AM-PAC $\leq 43.63$ (92\% vs 66\%, $\left.\chi^{2}[1, N=1,354], 134.4 ; P<.001\right)$. For $11 \%$ ( $n=147$ ) of the high-mobility group, the patient was not discharged home but was sent to post-acute care. Reviewing these patient charts showed the reasons for discharge to postacute care were predominantly personal or social needs (eg, homelessness, need for 24-hour supervision with no family support, patient request) or medical needs (eg, intravenous antibiotics or new tubes, lines, drains, or medications requiring extra nursing support or management). Only $16 \%$ of patients in this group ( $n=23$ ) experienced deconditioning necessitating physical therapy consult during hospitalization, per their record.

Compared with patients with admission AM-PAC score $>43.63$, patients with admission AM-PAC $\leq 43.63$ had significantly different changes in mobility as measured by mean delta AM-PAC score (delta AM-PAC, -0.41 for AM-PAC $>43.63$ vs +5.69 for AM-PAC $\leq 43.63$; $\mathrm{t}(3097)=-20.3 ; P<.001)$ (Table 1). 
TABLE 2. Factors Predicting Discharge to Home

\begin{tabular}{|c|c|c|}
\hline $\begin{array}{l}\text { Participants } \\
(\mathrm{N}=3091)\end{array}$ & $\begin{array}{l}\text { Adjusted OR for } \\
\text { discharge to home } \\
(95 \% \mathrm{Cl})\end{array}$ & $P$ value \\
\hline \multicolumn{3}{|l|}{ Age groups } \\
\hline$<65 y$ & $2.40(1.99-2.90)$ & $<.001$ \\
\hline$\geq 65 y$ & \multicolumn{2}{|c|}{ Reference } \\
\hline \multicolumn{3}{|c|}{ Admission AM-PAC score } \\
\hline High $^{b}$ & $5.38(4.36-2.89)$ & $<.001$ \\
\hline Low & \multicolumn{2}{|c|}{ Reference } \\
\hline \multicolumn{3}{|l|}{ Sex } \\
\hline Female & $1.13(0.95-1.34)$ & .180 \\
\hline Male & \multicolumn{2}{|c|}{ Reference } \\
\hline
\end{tabular}

\begin{tabular}{l} 
Length of stay $0.91(0.89-0.92)<<.001$ \\
\hline "By multivariate logistic regression controlling for age and length of stay. The primary \\
outcome of "discharge to home" was defined as: home with no needs or services, \\
home with outpatient physical therapy, or home with home health physical therapy. \\
'AM-PAC cutoff for inappropriateness was determined by previous studies determining \\
this as a predictor for discharge to home: "high" $>43.63$ and "low" $\leq 43.63$. \\
Abbreviation: AM-PAC, Activity Measure-Post Acute Care.
\end{tabular}

In multivariate logistic regression, AM-PAC $>43.63(\mathrm{OR}, 5.38$; $95 \% \mathrm{Cl}, 4.36-2.89 ; P<.001)$ and age younger than 65 years (OR, 2.40; 95\% Cl, 1.99-2.90; $P<.001$ ) were associated with increased odds of discharge to home (Table 2).

\section{DISCUSSION}

In this study, we found that physical therapists may be unnecessarily consulted on direct care hospitalist services as much as $38 \%$ of the time based on AM-PAC score. We also demonstrated that patients admitted with high mobility by AM-PAC score are more than five times as likely to be discharged to home. When admitted with high AM-PAC scores, patients had virtually no change in mobility during hospitalization, whereas patients with low AM-PAC scores gained mobility during hospitalization, underscoring the benefit of physical therapy referrals for this group.

Given resource scarcity and cost, achieving optimal physical therapy utilization is an important goal for healthcare systems. ${ }^{10}$ Appropriate allocation of physical therapy has the potential to improve outcomes from the patient to the payor level. While it may be necessary to consult physical therapy for reasons other than mobility later in the hospitalization, identifying patients who will benefit from skilled physical therapy at the time of admission can help prevent disability and institutionalization and shorten length of stay. ${ }^{5,6}$ Likewise, decreasing physical therapy referrals for low-risk patients can increase the amount of time spent rehabilitating at-risk patients.

There are limitations of our study worth considering. First, our analyses did not consider whether physical therapy contributed to patients' ability to return home after discharge. However, in our hospital, patients with AM-PAC $>43.63$ who cannot safely ambulate independently do progressive mobility with nursing staff. Our physical therapy leadership has also observed that the vast majority of highly mobile patients who are referred for physical therapy ultimately receive no treatment. Second, we did not consider discharge diagnosis, but our patient populations present with a wide variety of conditions, and it is impossible to predict their discharge diagnosis. By not including discharge diagnosis, we assess how AM-PAC performs on admission regardless of the medical condition for which someone is treated. Our hospital treats a high proportion of African American and a low proportion of White, Hispanic, and Asian American patients, limiting the generalizability of our findings. Although the AM-PAC "6-Clicks" score has been shown to have high interrater reliability among physical therapists, our AM-PAC scores are assessed and documented by our nursing staff, which might decrease accuracy. However, one single-center study noted an intraclass correlation coefficient of 0.96 between nurses and physical therapists for the AM-PAC "6-Clicks." "11

Despite these limitations, this study underscores the need to be more judicious in the decision to refer a patient for inpatient physical therapy, especially at the time of admission, and demonstrates the utility of using standardized mobility assessment to help in that decision-making process.

Disclosures: The authors reported no conflicts of interest.

Funding: Dr Arora is funded by the National Heart, Lung, and Blood Institute (NHLBI Grant K24HL136859).

\section{References}

1. Fazio S, Stocking J, Kuhn B, et al. How much do hospitalized adults move? A systematic review and meta-analysis. Appl Nurs Res. 2020;51:151189. https:// doi.org/10.1016/j.apnr.2019.151189

2. Brown CJ, Redden DT, Flood KL, Allman RM. The underrecognized epidemic of low mobility during hospitalization of older adults. J Am Geriatr Soc. 2009;57(9):1660-1665. https://doi.org/10.1111/j.1532-5415.2009.02393.x

3. Brown C.J, Friedkin RJ, Inouye SK. Prevalence and outcomes of low mobility in hospitalized older patients. J Am Geriatr Soc. 2004;52:1263-1270. https:// doi.org/10.1111/j.1532-5415.2004.52354.x

4. Zisberg A, Shadmi E, Gur-Yaish N, Tonkikh O, Sinoff G. Hospital-associated functional decline: the role of hospitalization processes beyond individual risk factors. J Am Geriatr Soc. 2015;63:55-62. https://doi.org/10.1111/ jgs.13193

5. Jette DU, Stilphen M, Ranganathan VK, Passek SD, Frost FS, Jette AM. Validity of the AM-PAC "6-Clicks" inpatient daily activity and basic mobility short forms. Phys Ther. 2014;94(3):379-391. https://doi.org/10.2522/ptj.20130199

6. Jette DU, Stilphen M, Ranganathan VK, Passek SD, Frost FS, Jette AM. AMPAC "6-Clicks" functional assessment scores predict acute care hospital discharge destination. Phys Ther. 2014;94(9):1252-1261. https://doi.org/10.2522/ ptj.20130359

7. Probasco JC, Lavezza A, Cassell A, et al. Choosing wisely together: physical and occupational therapy consultation for acute neurology inpatients. Neurohospitalist. 2018;8(2):53-59. https://doi.org/10.1177/1941874417729981

8. Young DL, Colantuoni E, Friedman LA, et al. Prediction of disposition within 48 hours of hospital admission using patient mobility scores. $J$ Hosp Med. 2020;15(9);540-543. https://doi.org/10.12788/jhm.3332

9. Jette DU, Stilphen M, Ranganathan VK, Passek S, Frost FS, Jette AM. Interrater reliability of AM-PAC "6-Clicks" basic mobility and daily activity short forms. Phys Ther. 2015;95(5):758-766. https://doi.org/10.2522/ptj.20140174

10. Juneau A, Bolduc A, Nguyen $P$, et al. Feasibility of implementing an exercise program in a geriatric assessment unit: the SPRINT program. Can Geriatr J. 2018;21(3):284-289. https://doi.org/10.5770/cgj.21.311

11. Hoyer EH, Young DL, Klein LM, et al. Toward a common language for measuring patient mobility in the hospital: reliability and construct validity of interprofessional mobility measures. Phys Ther. 2018;98(2):133-142. https:// doi.org/10.1093/ptj/pzx110 